\title{
Antimicrobial Activity of Antibiotic Producing Streptomyces macrosporus
}

\author{
E. Jennifer Emelda ${ }^{1}$, N.Vijayalakshmi ${ }^{2}$, T.Santhanakrishnan ${ }^{3}$ \\ ${ }^{1,2}$ Department of Microbiology, Sri Ramakrishna College of Arts \& Science for Women, Coimbatore, \\ Tamilnadu, India. \\ ${ }^{3}$ Centre for Bioscience and Nanoscience research centre, Coimbatore, Tamilnadu,India.
}

\begin{abstract}
The aim of the present study was to isolate and to identify the actinomycetes having antimicrobial activity. Actinomycetes strain isolated from soil samples collected at the Coimbatore region, Tamilnadu, India. Actinomycetes were isolated by spread plate technique and screening was done by parallel streak \& agar well diffusion method. Upon primary screening by parallel streak method out of 180 actinomycetes isolates around 25 isolates showed antimicrobial activity against test organisms. On secondary screening out of 25 isolates, 9 isolates showed activity. According to antimicrobial activity and spectrum broadness strain 7 was selected for further study. Based on the morphological, physiological, biochemical and phylogenetic characterization the strain 7 was identified as Streptomyces macrosporus. In conclusion the isolated strain has broad spectrum of antimicrobial activity against the test organisms.
\end{abstract}

\section{Introduction}

Search for new antibiotics effective against multidrug resistant pathogenic bacteria is presently an important area of antibiotic research. Natural products having novel structures have been observed to possess useful biological activities, soil is a natural reservoir for microorganisms and their antimicrobial products (1). Of all known drugs $70 \%$ have been isolated from actinomycetes of which $60 \%$ are used in medicine \& agriculture respectively. Streptomyces spp produce about $75 \%$ of commercially and medically useful antibiotics that are natural in origin such as erythromycin, Neomycin, tetracycline and Cefoxitin. Microorganisms constitute an in exhaustible reservoir of compounds with pharmacological, physiological, medical (or) agricultural applications.

\section{Materials \& Methods}

This study was carried out during September 2010 to August 2011 in the Department of microbiology, Sri Ramakrishna College of Arts \& Science for women, Coimbatore, TamilNadu, India.

Isolation of actinomycetes: Soil samples were collected from in and around areas of Coimbatore, TamilNadu, India. Nearly $1 \mathrm{~kg}$ of representative surface soil was collected from each of the areas. About $5 \mathrm{~g}$ of soil was taken and mixed with calcium carbonate and air dried at $20^{\circ} \mathrm{c}$ for 3 days. Actinomycetes were isolated by spread plate technique on starch casein nitrate agar and incubated at room temperature for 3- 4 days. Plates containing cyclohexamide at concentration of $50 \mu \mathrm{g} / \mathrm{ml}$ of medium (12).

Test culture: The MTCC cultures used for the test were Staphylococcus aureus 96, Staphylococcus aureus 2940, Escherichia coli 739, Candida albicans 237, Pseudomonas aeroginosa 2453 and Bacillus megaterium 287. The hospital cultures used for the test were Staphylococcus spp, E.coli, Bacillus spp, Candida spp, Aspergillus spp, Salmonella spp, Shigella spp and streptococcus spp. All the cultures were obtained from clinical laboratory and confirmed by standard biochemical test.

Screening of actinomycetes for antimicrobial activity: The screening method consist of two steps, Primary and secondary screening. Primary screening of actinomycetes isolates was done by parallel streak method (3) on nutrient agar (NA) for bacterial strains and Sabouraud's dextrose agar (SDA) for fungal \& yeast strains. Secondary screening of actinomycetes isolates was done by agar cup assay methods on Muller Hinton agar (MHA). The isolates possessing broad spectrum antimicrobial activity were processed further.

\section{Phenotypic characterization}

Aerial mass color and reverse side pigments: The mature sporulated aerial and substrate mycelium color was recorded in Glycerol asparagine agar (ISP 5), Inorganic salt starch agar (ISP 4), Oat meal agar (ISP 3), Tyrosine agar (ISP 7), Yeast extract malt extract agar (ISP 2), Glucose asparagine agar, Czapek agar with peptone, Czapek's agar \& Bennett's agar (2). The reverse side pigment of the colony was tested. Production of Melanoid pigments was tested on ISP 1 medium. 
Spore chain morphology: The spore bearing hyphae and spore chain was determined by direct examination of culture under microscope 1000x magnification by coverslip method using a well grown sporulated culture plate.

Physiological \& Biochemical characterization: The ability of the isolate to utilize degradation activities was checked with cellulose, Carboxy methyl cellulose, casein, tyrosine, gelatin and starch. Growth in presence of inhibitor compounds \& acid production from sugars are studied. Carbon and nitrogen sources were studied by the method recommended in International Streptomyces project. Carbon sources like glucose, Mannitol, arabinose, inositol, lactose, mannose, fructose, galactose, rhamnose, sucrose and xylose were tested on carbon utilization agar (ISP 9) supplemented with $1 \%$ carbon sources (10). The ability of the isolate to utilize various nitrogen sources like ammonium chloride, ammonium sulfate, L-asparagine, cystine, phenyl alanine, potassium nitrate, sodium nitrate and sodium nitrite for the growth also tested (11).

Fermentation and Preparation of crude extract: The isolates possessing broad spectrum antimicrobial activity in both primary and secondary screening were selected for fermentation. Fermentation was carried out by the submerged state culture in Erlenmeyer flask $(250 \mathrm{ml})$. The selected isolates was inoculated into starch casein nitrate broth and incubated at $28^{\circ} \mathrm{c}$ in orbital shaker (200-250 rpm) for 10 days. After incubation the broths were centrifuged at 10,000 rpm for $15 \mathrm{mins}$ and the cell free supernatant was filtered through Whattman No. 1 filter paper. The filtrate was transferred aseptically into a conical flask and stored at $4^{\circ} \mathrm{c}$ for further assay. The filtrate was subjected for solvent extraction method to recover antimicrobial metabolites in pure form (5). Hexane was added to the filtrate in the ratio of $1: 1(\mathrm{v} / \mathrm{v})$ and shaken vigorously for $1 \mathrm{~h}$ for complete extraction. The phase having antimicrobial property was poured in porcelain basin for evaporation. It was evaporated using flash evaporator and the residue obtained was weighed and dissolved in phosphate buffer for preservation. Other organic solvents like n-butanol, chloroform, acetone and ethyl acetate were also used to extract the antimicrobial metabolites by the same procedure. All the extracts were assayed for antimicrobial activity using respect solvent as control by agar cup assay method.

\section{$16 S$ rDNA sequencing}

Molecular taxonomy, sequencing and phylogenetic analysis: The genomic DNA isolation of actinomycetes was done by the method described by (4) and amplified by PCR using master mix kit medox mix (Medox, India). PCR conditions, the primers and methodology for sequencing were adapted from (6 \& 7). The sequencing was carried out in both sense and anti sense direction. The similarity and homology of the 16srDNA partial gene sequence was analyzed with the similar existing sequences available in the data bank of NCBI using BLAST search. The DNA sequences were aligned and phylogenetic tree was constructed by neighbor joining method using weighbor tree software. A bootstrap analysis of 100 replicates was carried out.

\section{Results}

180 different types of actinomycetes were isolated based on differences in colony morphology.

Screening of isolated actinomycetes for antimicrobial activity: Totally 180 actinomycetes isolates were screened for antimicrobial activity. Upon primary screening 25 isolates showed antimicrobial activity against 1 or more test organisms. Among the 25 isolates that were subjected for secondary screening only 9 isolates showed activity against test organisms. Actinomycete isolates namely Strain7 showed broad spectrum antimicrobial activity against all test organisms inhibiting growth \& forms a zone of lysis by cup assay method. Strain 7 were selected and characterized for further study (Table 1).

Phenotypic characterization: The cultural, morphological, physiological and biochemical characteristics of the isolate in different media are given in the (Table $2 \& 3$ ).

Solvent extraction: Different organic solvents like n- hexane, acetone, chloroform, ethyl acetate and n- butanol were tested for the extraction of the antibiotic. In case of the isolate S7, the antibiotic yield was observed in the residue using hexane. The residues obtained were light grey color and had flaky consistency.

16S rDNA sequencing: The blast search of $16 \mathrm{~S}$ rDNA sequence (585 base pairs) of the isolate showed maximum (98\%) similarity with Streptomyces macrosporus (EU301835) and the phylogenetic tree was constructed with bootstrap values (Figure 1). 
Antimicrobial activity of antibiotic producing Streptomyces macrosporus

Table 1 - Antimicrobial activity of the Streptomyces macrosporus

\begin{tabular}{lc}
\hline Microbial pathogens & Zone of inhibition (mm) \\
\hline MTCC STRAINS & Secondary screening-by cup assay method \\
\hline Escherichia coli -739 & 17 \\
Candida -227 & 23 \\
Staphylococcus aureus -96 & 14 \\
Pseudomonas aeroginosa -2453 & 21 \\
Bacillus megaterium -287 & \\
& \\
HOSPITAL PATHOGENS & 15 \\
Staphylococcus spp & 17 \\
Shigella spp & 15 \\
Streptococcus spp & 15 \\
Proteus spp & 16 \\
Escherichia coli & 14 \\
Salmonella spp & 17 \\
Klebsiella spp & 18 \\
Candida spp & 15 \\
Aspergillus spp &
\end{tabular}

Table 2 - Cultural characteristic of Streptomyces macrosporus on differential media

\begin{tabular}{lccc}
\hline Media & Growth & Color of aerial \& substrate mycelia & Pigmentation \\
\hline Yeast extract & - & - & - \\
Malt extract agar & Good & White \& Rose & - \\
Oat Meal agar & Good & Pale white \& pink & - \\
Bennets agar & Good & White \& Rose & - \\
Glucose asparagine agar & Good & Pale white and orange & - \\
Capek's agar & Good & White \& White & - \\
With peptone & & & - \\
Starch casein agar & Good & White \& pink & - \\
Capek's agar & Moderate & White \& white & \\
\hline
\end{tabular}

Table 3 - physiological \& Biochemical Characteristics Streptomyces macrosporus

Gram stain

Aerial \& substrate mycelium

Colony color

Melanin production

DEGRADATION ACTIVITY

Carboxy methyl cellulose, Cellulose, Starch \& casein

Gelatin, Tyrosine, Catalase, Oxidase \& $\mathrm{H}_{2}$ s production

CARBON SOURCES (1\% W/V)

D-Glucose, Sucrose, Mannose, Maltose, Mannitol

Inositol, Lactose, Fructose \& L-Rhamnose

D-Galactose, Arabinose \& Xylose

NITROGEN SOURCES $(1 \% \mathrm{~W} / \mathrm{V})$

Ammonium chloride, Ammonium sulfate, potassium nitrate

Sodium nitrate, L-Cystine \& L-asparagine

Sodium nitrite

GROWTH IN PRESENCE OF INHIBITOR COMPOUNDS

Potassium cyanide $(0.02 \%)$ \& Sodium chloride $(1 \%)$

Phenol $(0.1 \%) \&$ Sodium azide $(0.1 \%)$

ACID \& GAS PRODUCTION FROM SUGARS

Arabinose, Fructose, Galactose, Glucose, Inositol

Lactose, Mannitol, Maltose, Mannose, Rhamnose

Sucrose \& Xylose
$+$

White \& pink pinkish white

$+++$

$++$

$+++$

$++$

$+$

$-$

$\mathrm{A}+\mathrm{G}$

Note: ++ Good growth; +++ Moderate growth; A+G Acid \& Gas production 
Figure 1: Neighbor joining tree based on 16s rDNA sequences

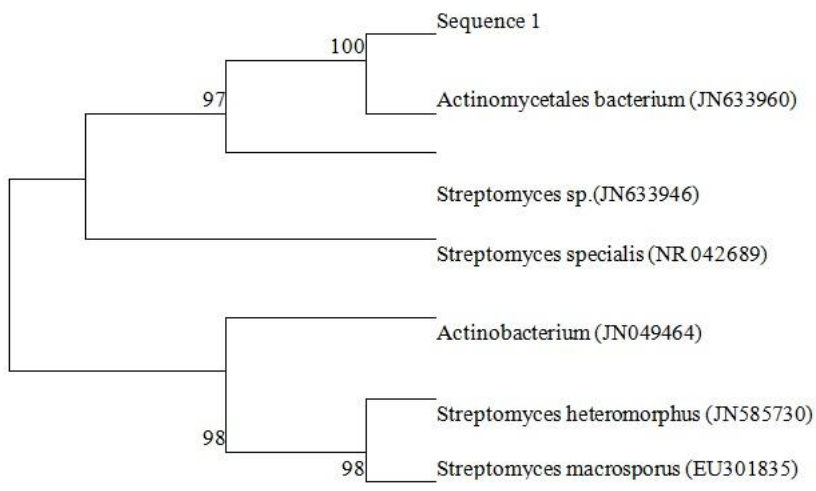

\section{Discussion}

Traditional methods of actinomycetes taxonomy relied heavily upon morphological, biochemical and physiological characteristics. Molecular techniques involving ribosomal RNA sequence analysis are commonly used to investigate evolutionary relationships within different genera of bacteria. In a routine antibiotic screening strategy, the strains producing interesting metabolites in screening are usually identified up to species level. Streptomyces strains which exhibited interesting results in primary screening and secondary screening were identified by $16 \mathrm{~s}$ rDNA gene sequencing. The morphological, biochemical and physiological characterization of these strains strongly suggested that they are the members of genus Streptomyces. The strains exhibited genetic similarity with genus and species of the Streptomyces macrosporus with the cases similarity up to $98 \%$ was found. It has been demonstrated that gene sequence data of an individual strain with a nearest neighbor exhibiting a similarity score of $<97 \%$ represents a new species, the meaning of similarity scores of $>97 \%$ is not as clear (8). The wide spectrum of microbiosis exhibited by cell free extracts obtained with hexane showed that Streptomyces macrosporus S7 is antagonistic actinomycetes. The compounds present within this extract showed highest activity against Staphylococcus aureus, Escherichia coli, Bacillus megaterium and Candida spp. The chemical and molecular characterization of S7 strain may pave the way for identification of new chemical entity against the pathogens.

\section{Acknowledgements}

We are very grateful to Mr. Babu- Sri Ramakrishna hospital, Coimbatore \& faculties of Microbiology Department, Sri Ramkrishna women's college for arts \& science, Coimbatore. Our sincere thanks to centre for bioscience and nanoscience research centre, Coimbatore.

\section{References}

[1]. Dancer., 2004. How antibiotics can make us sick: the less obvious adverse effects of antimicrobial chemotherapy. The lancet infectious diseases. $4: 611-619$.

[2]. Das,s P.s lyla 2008.Charecterization and identification of actinomycetes existing systems,complexities and future directions.Natl.Acad.sci.lett.,31:149-160.

[3]. Egorov. N., 1995. Isolation of microorganisms producing antibiotics and their biological activity. Antibiotics a scientific approach. 1st (edn). 132-137

[4]. Sambrook J., Fritsch E.F., Maniatis T. (1989) Molecular cloning: A laboratory manual (Cold Spring Harbor Laboratory Press, Cold Spring Harbor, NY), 2nd ed

[5]. Liu, C.M ., J.W. Westley, TE. Herman, B.L.T. prasser N. palleroni, R.H evans and P.H miller 1986. Novel polyether antibiotics. X14873 A,G and H produced by streptomyces. Taxonomy of the producing culture, fermentation, biological and inosphores properties of antibiotics. Journal of antibiotics 39 (12): $1712-1718$

[6]. Magarvey,N.a 2004.Isolation and characterization of novel marine derived actinomycetes taxa rich in bioactive metabolites.Appl.Environ.Microbiol.,70:7520-7529.

[7]. Mincer T.J., 2002.widwspread and persistant populations of a major new marine actinomycetes taxon in ocean sediments.Appled environ.

[8]. Petti, C. A. (2007). Detection and identification of microorganism gene amplification and sequencing. Clin Infect Dis 44: 11081114.

[9]. Smith.JE., 1989. Perspective in biotechnology and applied microbiology. Murray Moo Young, 105-134

[10]. Shirling, E. and D. Gottlieb, 1966. Methods for characterization of Streptomyces species. Intl. j. syst. Bacterial. 16: 313-340.

[11]. Williams. ST, M.Good fellow, G.Alderson, Wellington, Sneath, MJ. 1983. Numerical classification of Streptomyces and related genera. Journal of General Microbiology 129:1743-1813.

[12]. Williams S.T and F.L Davis 1965. Use of antibiotics for selective isolation and Enumeration of actinomycetes in soil. Journal of general microbiology 38:251-261 WHO 2002. Death by cause, sex and ortality stratum in WHO region, estimates for 2001. World Health Report, world health organization. 